\title{
Considerations in imaging interpretations for colitis in critically ill patients during the COVID-19 era
}

\author{
Sooyoung Martin ${ }^{1}$ (D ) Jonathan Pierce ${ }^{1} \cdot$ Elias G. Kikano ${ }^{1} \cdot{\text { Derek } \operatorname{Vos}^{1} \cdot \text { Sree Harsha Tirumani }}^{1} \cdot$ Nikhil Ramaiya $^{1}$
}

Received: 14 November 2020 / Accepted: 8 March 2021 / Published online: 17 March 2021

(C) American Society of Emergency Radiology 2021

\begin{abstract}
Objective The study aims to demonstrate risk factors for colitis in intensive care unit patients with and without coronavirus disease 2019 (COVID-19).

Methods Retrospective review was performed to identify intensive care unit (ICU) patients with the diagnosis of COVID-19 with computed tomography (CT) between March 20 and December 31, 2020. ICU patients without COVID-19 diagnosis with CT between March 20 and May 10, 2020 were also identified. CT image findings of colitis or terminal ileitis as well as supportive treatment including ventilator, vasopressors, or extracorporeal membrane oxygenation (ECMO) were recorded. Statistical analysis was performed to determine if clinical factors differed in patients with and without positive CT finding.

Results Total 61 ICU patients were selected, including 32 (52\%) COVID-19-positive patients and 29 (48\%) non-COVID-19 patients. CT findings of colitis or terminal ileitis were identified in 27 patients $(44 \%)$. Seventy-four percent of the patients with positive CT findings (20/27) received supportive therapies prior to CT, while $56 \%$ of the patients without abnormal CT findings (19/34) received supportive therapies. Vasopressor treatment was significantly associated with development of colitis and/or terminal ileitis $(p=0.04)$ and COVID-19 status was not significantly different between these groups $(p=0.07)$.

Conclusions In our study, there was significant correlation between prior vasopressor therapy and imaging findings of colitis or terminal ileitis in ICU patients, independent of COVID-19 status. Our observation raises a possibility that the reported COVID19-related severe gastrointestinal complications and potential poor outcome could have been confounded by underlying severe critically ill status, and warrants a caution in diagnosis of gastrointestinal complication.
\end{abstract}

Keywords Coronavirus disease 2019 · Colitis · Critical illness · Multi-detector computed tomography

\section{Introduction}

In December 2019, an unknown pneumonia initially broke out clustered in the region of Wuhan, China. The source organism identified as severe acute respiratory syndrome novel coronavirus 2 (SARS-nCOV-2) rapidly spread worldwide leading to World Health Organization's (WHO) declaration of pandemic status in March 2020. Coronavirus disease 2019 (COVID-19) has resulted in a high degree of morbidity and mortality, having surpassed 2.5 million deaths with the total confirmed cases

Sooyoung Martin

Sooyoung.martin@UHhospitals.org

1 Department of Radiology, University Hospitals Cleveland Medical Center, 11100 Euclid Avenue, Cleveland, OH 44106, USA of more than 114.8 million worldwide as of March 2021 according to WHO [1].

Although the majority of patients present with respiratory symptoms and signs, COVID-19 patients also experience symptoms involving other organ systems, including the central nervous system, cardiovascular, hematologic, and urinary system [2]. Varying degrees of gastrointestinal (GI) symptoms are commonly presented by COVID-19 patients, ranging from mild but more frequent nausea or vomiting to more serious conditions such as ischemic colitis which may be detectable on imaging studies or colonoscopy [3]. In addition, there have been reports suggesting the potential impact of COVID-19 status on severe GI complication resulting in poor clinical outcomes [4]. However, the causal relationship between COVID-19 infection and severity of the GI complication has not been clarified. This is especially important in critically ill patients who tend to have multiple comorbidities and confounding clinical factors. In these cases of critically ill 
COVID-19 patients with GI imaging findings, clinical significance of the imaging findings and ultimate impact on management have not been established. Therefore, we have retrospectively reviewed and compared the radiologic findings of colitis, COVID-19 status, and severity of clinical status in the intensive care unit (ICU) setting.

\section{Methods}

This study was approved by institutional review board for retrospective review. Patients who were admitted to the ICU with a COVID-19 diagnosis within a single healthcare system who also received computed tomography (CT) imaging of the abdomen and pelvis between March 20 and December 31, 2020 were retrospectively identified. For comparison purpose, patients without a COVID-19 diagnosis admitted in ICU with at least one $\mathrm{CT}$ of the abdomen and pelvis obtained between March 20 and May 10, 2020 were retrospectively identified as well.

$\mathrm{CT}$ examinations of these patients were reviewed by two radiologists (one reader with four years of experience and the other reader with 23 years of experience), blinded for the patients' COVID-19 status or other clinical factors. Patients with secondary bowel changes explainable by other underlying conditions were excluded to minimize confusion from reactive changes. These include recent post-surgical changes in the abdomen and pelvis, generalized increased volume status, a mass involving the bowel with focal fat stranding, localized fat stranding due to inflammation or infection in the adjacent organs, or intraabdominal bleeding. Imaging findings suggestive of colitis or terminal ileitis including bowel wall thickening, colonic distension/luminal fluid, pneumatosis, and pneumoperitoneum were documented.

Retrospective review of the medical record was conducted to identify demographic data and clinical factors, including COVID-19 test positivity (either positive COVID-19 PCR test during admission or documented outside positive COVID-19 test on admission note), history of intubation with mechanical ventilation, vasopressor support, or extracorporeal membrane oxygenation (ECMO) usage within 30 days prior to the $\mathrm{CT}$. Patients' clinical outcome and disposition at discharge were also documented.

Statistical analysis with unpaired $t$-test was performed to compare age between patients with colitis and without colitis. Statistical analysis with Fisher's exact test was performed to determine if patients with and without colitis differed in terms of gender, COVID-19 status, ECMO, vasopressor, and ventilator treatment. All $p$ values were based on a two-sided hypothesis. A $p$ value of $<0.05$ was considered to be statistically significant. Statistical analyses were conducted using JMP® Software (JMP® PRO version 15.0.0, SAS Institute Inc., Cary, NC, 1989-2007).

\section{Results}

A total of 46 COVID-19-positive ICU patients were identified to have received at least one $\mathrm{CT}$ abdomen and pelvis between March and December 2020. These patients included 42 patients with positive COVID-19 PCR test during admission as well as four patients with documented outside positive COVID-19 test on admission note. Additionally, a total of 65 non-COVID-19 ICU patients were identified to have received at least one $\mathrm{CT}$ abdomen and pelvis exam between March and May 2020. Out of the 111 total patients, 50 patients were excluded due to other confounding factors (postoperative status, $n=19$; volume overload, $n=12$; malignancy involving GI tract, $n=3$; adjacent organ infection/inflammation, $n=5$; adjacent intraabdominal bleeding, $n=11$ ) (Fig. 1). Retrospective review of the medical record was performed for the remaining $61 \mathrm{ICU}$ patients including COVID-19-positive patients $(n=32)$ and non-COVID-19 patients $(n=29)$. The average age of total $61 \mathrm{ICU}$ patient was 61.2 years old, with $62 \%$ ( $n$ = 39) male and 38\% ( $n=23)$ female (Table 1).

Among those 61 patients, 27 patients $(44 \%)$ had positive CT findings for colitis or terminal ileitis $(n=26$ for colitis, $n=1$ for terminal ileitis) and 34 patients $(56 \%)$ did not have CT findings suggestive of colitis or terminal ileitis (Fig. 2). Among 27 patients with positive CT findings for colitis or terminal ileitis, 18 patients $(67 \%)$ were positive for COVID-19 and 20 patients $(74 \%)$ received advanced critical care supportive therapy prior to CT. These advanced critical care supportive therapies include ECMO $(n=2)$, ventilator $(n=$ $16)$, and vasopressor treatment $(n=19)$. Among 34 patients without CT colitis findings, 14 patients $(41 \%)$ were positive for COVID-19 and 19 patients (56\%) received advanced critical care supportive therapy prior to $\mathrm{CT}$, including $\mathrm{ECMO}(n=1)$, ventilator $(n=17)$, and vasopressor treatment $(n=14)$.

Only recent history of vasopressor treatment was significantly different between patients with positive CT findings and patients without $\mathrm{CT}$ findings suggestive of colitis and/or terminal enteritis $(p=0.04)$. Other variables including COVID-19 status, recent history of ECMO, and mechanical ventilation did not show statistically significant difference between patients with and without CT findings of colitis based on two-tailed analysis (Table 1).

Within the subgroup of CT findings suggestive of colitis and/or terminal enteritis $(n=27), 50 \%$ of patients with positive COVID-19 test (9/18) had poor outcome (death in seven patients; hospice in two patients) and 56\% of non-COVID-19 patients $(5 / 9)$ had poor outcome (death in three patients, hospice in one patient, and colectomy due to ischemic colitis in one patient) (Fig. 3). 
Table 1 Patient demographic data and clinical information with and without CT findings of colitis or terminal ileitis during ICU admission

\begin{tabular}{|c|c|c|c|c|}
\hline & $\begin{array}{l}\text { ICU patients with } \\
\text { CT }(n=61)\end{array}$ & $\begin{array}{l}\text { Positive CT findings for colitis } \\
\text { or terminal ileitis }(n=27)\end{array}$ & $\begin{array}{l}\text { Negative CT findings for colitis } \\
\text { or terminal ileitis }(n=34)\end{array}$ & $p$ value \\
\hline \multicolumn{5}{|l|}{ Demographic data } \\
\hline Age (mean, range) & $61.2(19-100)$ & $63.6(29-100)$ & $59.3(19-92)$ & 0.32 \\
\hline Gender (M: F) & $38: 23$ & $17: 10$ & $21: 13$ & 1 \\
\hline \multicolumn{5}{|l|}{ Clinical information } \\
\hline COVID-19 positive & 32 & $18(67 \%)$ & $14(41 \%)$ & 0.07 \\
\hline ECMO & 3 & $2(7 \%)$ & $1(3 \%)$ & 0.58 \\
\hline Ventilator & 33 & $16(59 \%)$ & $17(50 \%)$ & 0.61 \\
\hline Vasopressor & 33 & $19(70 \%)$ & $14(41 \%)$ & 0.04 \\
\hline
\end{tabular}

COVID-19 coronavirus disease 2019, ECMO extracorporeal membrane oxygenation

Of the COVID-19-positive patients ( $n=32), 18$ patients (56\%) showed findings suggestive of colitis and/or terminal ileitis (Fig. 4). Sixteen patients were on mechanical ventilation (nine patients with positive CT finding, seven patients without CT finding), 17 patients received vasopressor (12 patients with positive $\mathrm{CT}$ finding, five patients without $\mathrm{CT}$ finding), and none of these patients were on ECMO prior to imaging. None of these advanced critical care supportive treatments showed significant correlation with CT finding of colitis and/or terminal ileitis.

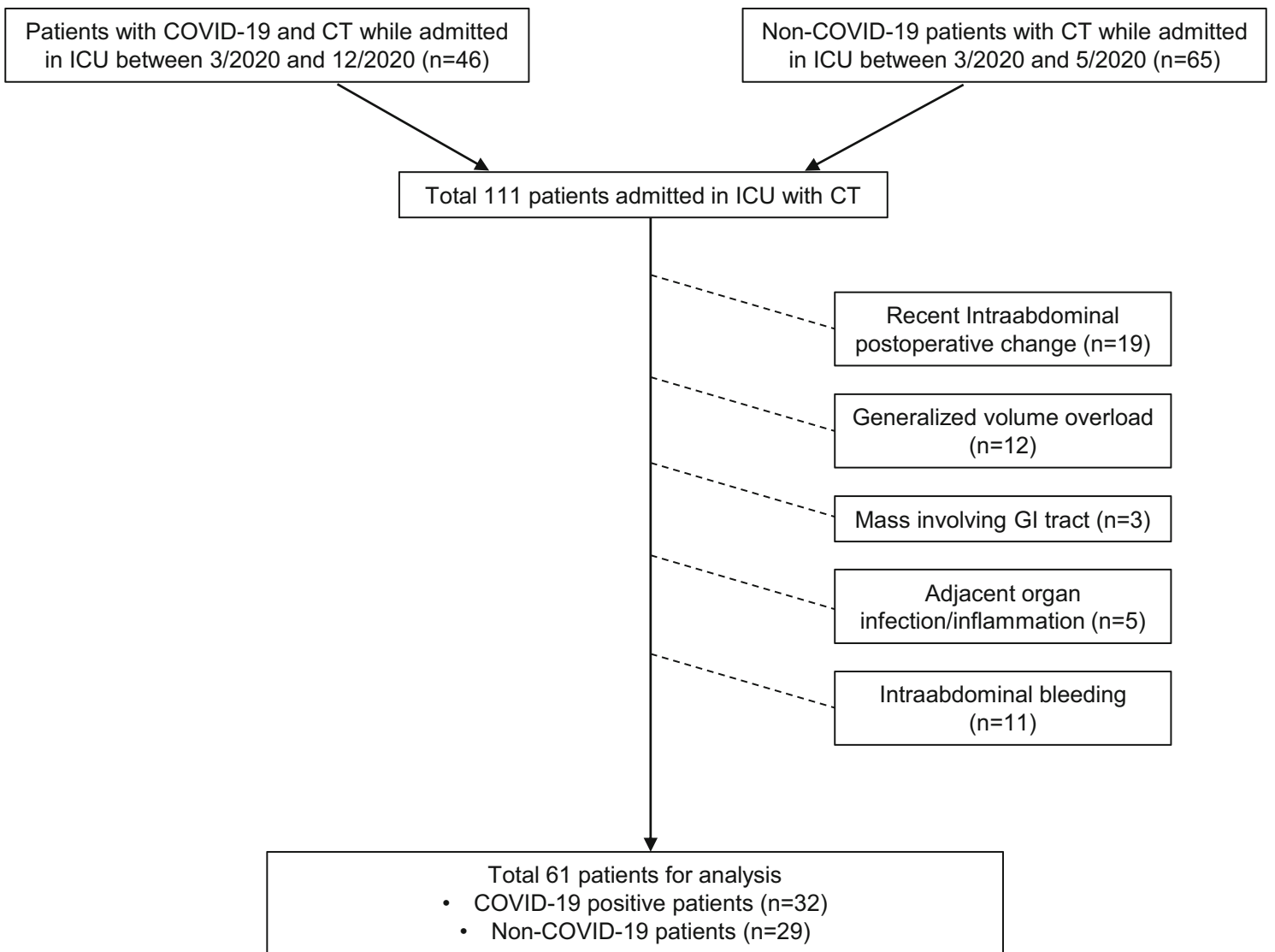

\section{Discussion}

As COVID-19 cases reported worldwide continues to increase, multi-organ involvement secondary to COVID-19 infection have been more frequently reported. As part of multisystemic manifestations, gastrointestinal symptoms varying from nausea, vomiting to ischemic colitis have been variably recognized $[3,5]$.

Several hypotheses have been raised to explain the involvement of GI system as an entry or transmission route given 


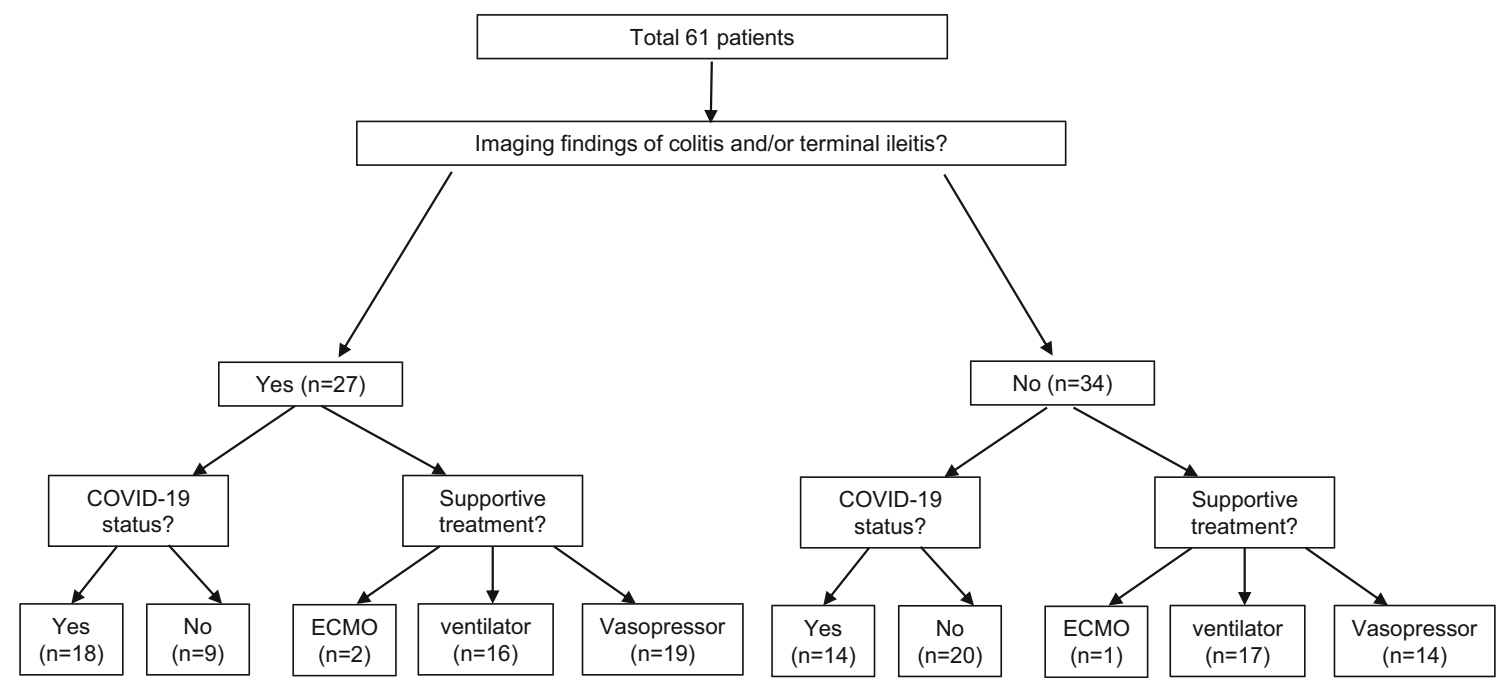

Fig. 2 Flow chart for patients with or without imaging findings suggestive of colitis or terminal ileitis

SARS-nCOV-2 RNA detection in stool samples [6]. These include via angiotensin-converting enzyme 2 (ACE2) receptor expressed throughout the intestinal epithelium [7], as part of systemic involvement secondary to heightened inflammatory reaction and cytokine storm [8], and hypercoagulability with micro-thrombosis [3].

Several studies have been published identifying GI complications on radiologic examinations in COVID-19 patients. In a study by Bhayana et al., $31 \%$ of abdominal CT images showed abnormal bowel wall findings and $20 \%$ of ICU patients' CT images had findings concerning for bowel ischemia requiring surgical management [3]. In case series by Norsa et al., seven patients with positive COVID-19 were diagnosed with potentially higher prevalence and higher mortality when compared to patients with bowel ischemia during preCOVID-19 period [4]. However, these studies have not specifically looked at bowel complications in the ICU setting.
A study by El Moheb et al. did focus on critically ill patients with and without COVID-19 and found higher percentage of overall GI complications (74\% vs $37 \%$ ) as well as higher rate of intestinal ischemia (4\% vs $0 \%$ ) in COVID-19positive patients compared with propensity score matched patients from a pre-COVID-19 time period [9]. This study demonstrated potentially higher bowel complication rate in the COVID-19 ICU population, but the severity of underlying critically ill status and its secondary effect have not been taken account. Therefore, whether or not COVID-19 positivity status itself was the major factor in bowel complication remains unclear.

Compared to the general population and patients with milder illness, the ICU patient population deserves special attention due to various complications, multi-factorial nature from multiple comorbidities, and the variety of supporting treatments which may further complicate and confound
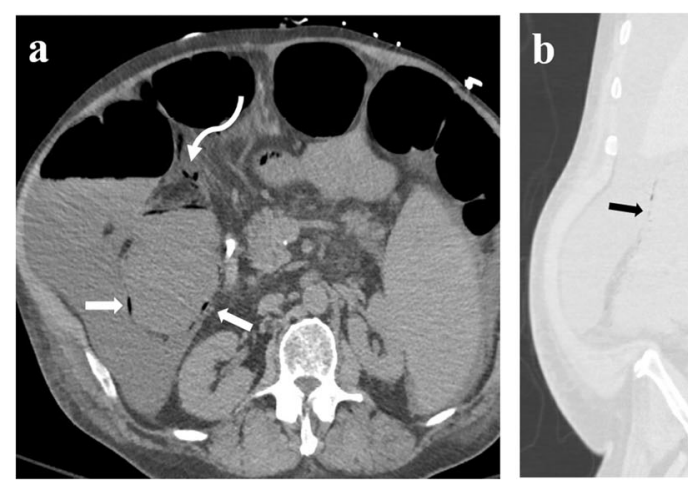

Fig. 3 Fifty-nine-year-old male obtained non-contrast CT of abdomen and pelvis for the concern of small-bowel obstruction, who was not diagnosed to have coronavirus disease 2019 prior to CT. Patient's history was notable for coronary artery bypass surgery 8 days ago and non-alcoholic steatohepatitis-induced liver cirrhosis. Axial (a) and coronal (b and c) CT images demonstrated fluid filled ascending colon

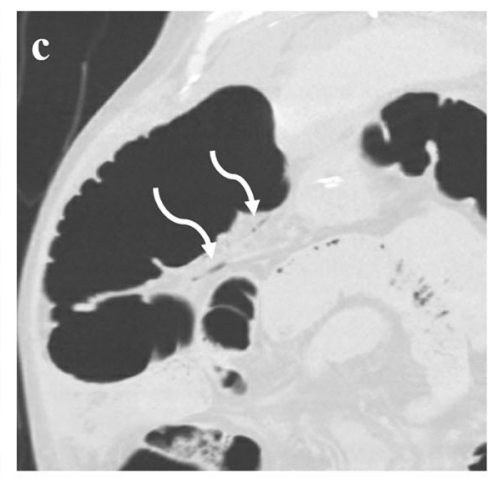

with pneumatosis (white straight arrow in $\mathbf{a}$, black straight arrow in $\mathbf{b}$ ), as well as linear branching air along the mesenteric vessels (white curved arrow in a and c), concerning for ischemic colitis. Subsequently, patient underwent extended right hemicolectomy and was confirmed to have transmural ischemia and necrosis of the ascending colon 


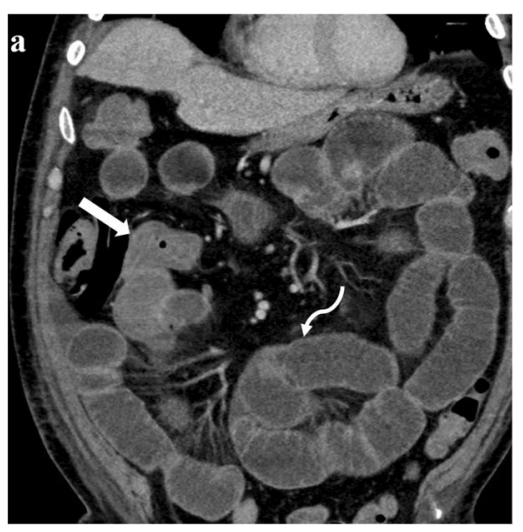

Fig. 4 Sixty-nine-year-old male admitted for coronavirus disease 2019 pneumonia with acute respiratory distress syndrome who received ventilation and vasopressor treatment. CT was obtained due to increasing abdominal distension. Coronal (a) and axial (b) images of contrast enhanced CT demonstrate diffuse wall thickening along the

underlying conditions. Critical illness and mechanical ventilator treatment statuses have been thought to contribute to gastrointestinal ischemic events via splanchnic hypoperfusion. Additionally, several parameters including ICU care, vasopressor support, and ventilator treatment were identified as risk factors for patients who failed initial non-operative management for ischemic colitis [10, 11].

In our study focusing on ICU patients with imaging findings of colitis and/or terminal ileitis at a single healthcare system during the COVID-19 pandemic, recent history of supportive treatment such as vasopressor showed correlation with positive image findings of colitis, while COVID-19 status was not significantly different between groups with and without CT finding of colitis. It was also notable that higher percentage of ICU patients with CT findings of colitis (20 out of 27 patients, $74 \%$ ) had received at least one of the supportive treatments, suggestive of the severity of underlying critical illness. In addition, comparable or slightly higher rate of poor outcome including ischemic colitis requiring surgical treatment, hospice care, or death was noted in non-COVID-19 patients with positive CT finding compared to COVID-19positive patients with positive $\mathrm{CT}$ finding, although outcome analysis was not performed due to small number of sample size. Based on our observation, we are raising questions that the etiology of bowel complications and its severity may not necessarily be due to COVID-19 status itself especially in ICU population, but the severity of critically ill status or co-existing clinical conditions may elicit or amplify the gastrointestinal complications. Furthermore, possibility of other etiologies to explain abnormal bowel findings on CT should be considered and correlated with clinical condition and medical history, before attributing it to COVID-19-related colitis when interpreting $\mathrm{CT}$ in this subset of patients. Our observation is also in line with the recently published study by Elmunzer et al., where GI symptoms were frequent complaints in

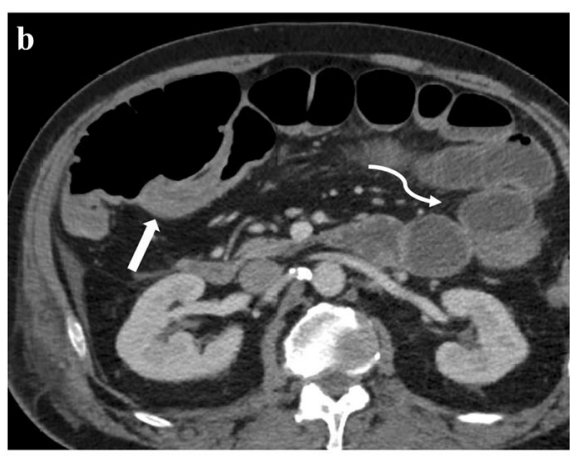

terminal ileum (straight arrows in $\mathbf{a}$ and $\mathbf{b}$ ) and mild diffuse dilatation of the fluid-filled proximal small-bowel loops (curved arrows in $\mathbf{a}$ and $\mathbf{b}$ ), suggestive of terminal ileitis and secondary bowel dilatation. Patient was conservatively managed and discharged approximately 2 weeks after

hospitalized COVID-19 patients, but not associated with severe outcome clinically [12].

Our study is limited by small sample size especially in COVID-19-positive subgroup analysis, as we have scrutinized and focused on only critically ill patients with positive imaging findings of terminal ileitis or colitis. Furthermore, we have used CT findings as a primary tool to assess colitis or terminal ileitis and could have underestimated the occurrence of the occult GI symptoms. However, our study is primarily to guide radiologists' interpretation of the $\mathrm{CT}$ finding in the clinical setting during the era of COVID-19 pandemic. Additionally, CT is used as a main modality in current practice setting to assess patients with acute abdominal symptoms, and we believe that in the appropriate clinical setting, CT is a useful tool to detect severe enterocolitis or ischemic colitis, which will more likely require aggressive management or at least close clinical monitoring [13].

Since pandemic status was officially declared in March 2020, there have been new cases of COVID-19 without a promising decline in numbers and it is expected that COVID-19 will continue to be a part of medical practice for the foreseeable future. In addition, approximately $30 \%$ of hospitalized COVID-19 patients ended up in critically ill status requiring ICU admission. This included intubation and/or ECMO treatment and previously reported studies identified as high as $29 \%$ of hospitalized patients experienced acute respiratory distress syndrome (ARDS) [14]. Therefore, further studies need to be conducted to verify the effect of critically ill status itself versus COVID-19 status on gastrointestinal complications in the ICU population, to help in the interpretation of imaging studies, and guide appropriate management in this COVID-19 era.

In conclusion, we believe that measured caution needs to be taken during interpretation of gastrointestinal manifestations in the setting of COVID-19, especially in critically ill 
patients, and other clinical factors must be considered as well before attributing the etiology of abnormal CT appearance of bowel solely to the COVID-19 status.

Availability of data and material Dataset from this study is available from the corresponding author.

\section{Declarations}

Ethics approval Approval was obtained from the institutional review board for retrospective review.

\section{Consent to participate Waived.}

Consent for publication Waived.

Conflict of interest The authors declare that they have no conflict of interest.

Code availability Not applicable.

Abbreviations $C T$, Computed tomography; COVID-19, Coronavirus disease 2019; ECMO, Extracorporeal membrane oxygenation; $G I$, Gastrointestinal; $I C U$, Intensive care unit; $S A R S-n C O V$-2, Severe acute respiratory syndrome novel coronavirus 2; WHO, World Health Organization

\section{References}

1. WHO Coronavirus Disease (COVID-19) Dashboard | WHO Coronavirus Disease (COVID-19) Dashboard. https://covid19. who.int/. Accessed 4 March 2021

2. Zheng KI, Feng G, Liu WY et al (2020) Extrapulmonary complications of COVID-19: a multisystem disease? J Med Virol

3. Bhayana R, Som A, Li MD, Carey DE, Anderson MA, Blake MA, Catalano O, Gee MS, Hahn PF, Harisinghani M, Kilcoyne A, Lee SI, Mojtahed A, Pandharipande PV, Pierce TT, Rosman DA, Saini S, Samir AE, Simeone JF, Gervais DA, Velmahos G, Misdraji J, Kambadakone A (2020) Abdominal imaging findings in COVID19: preliminary observations. Radiology. 297:E207-E215. https:// doi.org/10.1148/radiol.2020201908

4. Norsa L, Pietro B, Indriolo A et al (2020) Poor outcome of intestinal ischemic manifestations of COVID-19. Gastroenterology. 159: 1595-1597.e1. https://doi.org/10.1053/j.gastro.2020.06.041

5. Pan L, Mu M, Yang P et al (2020) Clinical characteristics of COVID-19 patients with digestive symptoms in Hubei, China: a descriptive, cross-sectional, multicenter study. Am J Gastroenterol. https://doi.org/10.14309/ajg.0000000000000620

6. Aroniadis OC, DiMaio CJ, Dixon RE, Elmunzer BJ, Kolb JM, Mendelsohn R, Ordiah CO, Rockey DC, Singal AG, Spitzer RL, Tierney WM, Wani S, Yadav D, North American Alliance for the Study of Digestive Manifestations of Covid-19 (2020) Current knowledge and research priorities in the digestive manifestations of COVID-19. Clin Gastroenterol Hepatol 18:1682-1684. https:// doi.org/10.1016/j.cgh.2020.04.039

7. Hoffmann M, Kleine-Weber H, Schroeder S, Krüger N, Herrler T, Erichsen S, Schiergens TS, Herrler G, Wu NH, Nitsche A, Müller MA, Drosten C, Pöhlmann S (2020) SARS-CoV-2 cell entry depends on ACE2 and TMPRSS2 and is blocked by a clinically proven protease inhibitor. Cell. 181:271-280.e8. https://doi.org/10. 1016/j.cell.2020.02.052

8. Mehta P, McAuley DF, Brown M, Sanchez E, Tattersall RS, Manson JJ, HLH Across Speciality Collaboration, UK (2020) COVID-19: consider cytokine storm syndromes and immunosuppression. Lancet 395:1033-1034

9. El Moheb M, Naar L, Christensen MA et al (2020) Gastrointestinal complications in critically ill patients with and without COVID-19. JAMA. 324:1899. https://doi.org/10.1001/jama.2020.19400

10. Mutlu GM, Mutlu EA, Factor P (2001) GI complications in patients receiving mechanical ventilation. Chest. 119:1222-1241. https:// doi.org/10.1378/chest.119.4.1222

11. Paterno F, McGillicuddy EA, Schuster KM, Longo WE (2010) Ischemic colitis: risk factors for eventual surgery. Am J Surg 200: 646-650. https://doi.org/10.1016/j.amjsurg.2010.07.005

12. Elmunzer BJ, Spitzer RL, Foster LD, Merchant AA, Howard EF, Patel VA, West MK, Qayed E, Nustas R, Zakaria A, Piper MS, Taylor JR, Jaza L, Forbes N, Chau M, Lara LF, Papachristou GI, Volk ML, Hilson LG, Zhou S, Kushnir VM, Lenyo AM, McLeod CG, Amin S, Kuftinec GN, Yadav D, Fox C, Kolb JM, Pawa S, Pawa R, Canakis A, Huang C, Jamil LH, Aneese AM, Glamour BK, Smith ZL, Hanley KA, Wood J, Patel HK, Shah JN, Agarunov E, Sethi A, Fogel EL, McNulty G, Haseeb A, Trieu JA, Dixon RE, Yang JY, Mendelsohn RB, Calo D, Aroniadis OC, LaComb JF, Scheiman JM, Sauer BG, Dang DT, Piraka CR, Shah ED, Pohl H, Tierney WM, Mitchell S, Condon A, Lenhart A, Dua KS, Kanagala VS, Kamal A, Singh VK, Pinto-Sanchez MI, Hutchinson JM, Kwon RS, Korsnes SJ, Singh H, Solati Z, Willingham FF, Yachimski PS, Conwell DL, Mosier E, Azab M, Patel A, Buxbaum J, Wani S, Chak A, Hosmer AE, Keswani RN, DiMaio CJ, Bronze MS, Muthusamy R, Canto MI, Gjeorgjievski VM, Imam Z, Odish F, Edhi AI, Orosey M, Tiwari A, Patwardhan S, Brown NG, Patel AA, Ordiah CO, Sloan IP, Cruz L, Koza CL, Okafor U, Hollander T, Furey N, Reykhart O, Zbib NH, Damianos JA, Esteban J, Hajidiacos N, Saul M, Mays M, Anderson G, Wood K, Mathews L, Diakova G, Caisse M, Wakefield L, Nitchie H, Waljee AK, Tang W, Zhang Y, Zhu J, Deshpande AR, Rockey DC, Alford TB, Durkalski V (2020) Digestive manifestations in patients hospitalized with COVID-19. Clin Gastroenterol Hepatol Off Clin Pract J Am Gastroenterol Assoc. https://doi.org/10.1016/j. cgh.2020.09.041

13. Dhatt HS, Behr SC, Miracle A, Wang ZJ, Yeh BM (2015) Radiological evaluation of bowel ischemia. Radiol Clin N Am 53:1241-1254

14. Greenland JR, Michelow MD, Wang L, London MJ (2020) COVID-19 infection: implications for perioperative and critical care physicians. Anesthesiology. 132:1346-1361. https://doi.org/ 10.1097/ALN.0000000000003303

Publisher's note Springer Nature remains neutral with regard to jurisdictional claims in published maps and institutional affiliations. 